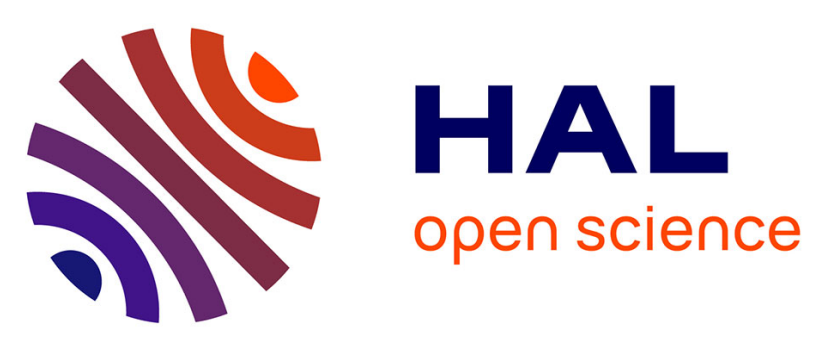

\title{
Nanometric emulsions encapsulating solid particles as alternative carriers for intracellular delivery
}

Sandrine Quignard, Ghislaine Frebourg, Yong Chen, Jacques Fattaccioli

\section{To cite this version:}

Sandrine Quignard, Ghislaine Frebourg, Yong Chen, Jacques Fattaccioli. Nanometric emulsions encapsulating solid particles as alternative carriers for intracellular delivery. Nanomedicine, 2016, 11 (16), pp.2059-2072. 10.2217/nnm-2016-0074 . hal-01536652

\section{HAL Id: hal-01536652 \\ https://hal.science/hal-01536652}

Submitted on 31 Aug 2021

HAL is a multi-disciplinary open access archive for the deposit and dissemination of scientific research documents, whether they are published or not. The documents may come from teaching and research institutions in France or abroad, or from public or private research centers.
L'archive ouverte pluridisciplinaire HAL, est destinée au dépôt et à la diffusion de documents scientifiques de niveau recherche, publiés ou non, émanant des établissements d'enseignement et de recherche français ou étrangers, des laboratoires publics ou privés. 


\section{Nanometric emulsions encapsulating solid particles as alternative carriers for intracellular delivery}

\begin{abstract}
Aim: Formulate nanometric oil droplets for encapsulating solid nanoparticles and assess their interactions with cells. Materials $\&$ methods: Soybean oil droplets, stabilized by Pluronic F68 surfactant, incorporating hydrophobically modified fluorescent silica, nanoparticles were obtained. Cytotoxicity over time, internalization, subsequent intracellular localization and internalization pathways were assessed by microscopy (fluoresence and TEM) in vitro with HeLa cells. Results: Oil droplets encapsulating solid nanoparticles are readily internalized by HeLa cells like free nanoparticles but the intracellular localization differs (nanoemulsions less colocalized with lysosomes) as well as internalization pathway is used (nanoemulsions partially internalized by nonendocytic transport). No cytotoxicity could be observed for either particles tested. Conclusion: Our results confirm that nanometric emulsions encapsulating solid nanoparticles can be used for alternative and multifunctional intracellular delivery.
\end{abstract}

Keywords: hybrid nanoparticles • intracellular delivery • passive internalization

\author{
Sandrine Quignard*,1,2,3, \\ Ghislaine Frébourg ${ }^{4}$, \\ Yong Chen ${ }^{1,2,3}$ \& Jacques \\ Fattaccioli $* *, 1,2,3$ \\ 'École Normale Supérieure - PSL Research \\ University, Département de Chimie, \\ 24 rue Lhomond, F-75005 Paris, France \\ ${ }^{2}$ Sorbonne Universités, UPMC Univ. Paris \\ 06, PASTEUR, F-75005 Paris, France \\ ${ }^{3}$ CNRS, UMR 8640 PASTEUR, F-75005 \\ Paris, France \\ ${ }^{4}$ Institut de Biologie Paris-Seine FR3631, \\ Service de Microscopie Electronique, \\ Université Pierre et Marie Curie, 9 Quai \\ Saint Bernard, 75005 Paris, France \\ *Author for correspondence: \\ sandrine.quignard@ens.fr \\ **Author for correspondence: \\ jacques.fattaccioli@ens.fr
}

One of the current challenges faced by medical science is to develop multifunctional carriers for efficient delivery of poorly watersoluble drugs. Indeed, approximately $40 \%$ of marketed drugs and more than $70 \%$ of promising drug candidates are insoluble in water which leads to a poor bioavailability that definitely compromises their therapeutic effects [1]. These hydrophobic drugs thus necessitate adequate carriers providing hydrophobicity to encapsulate the molecule and sufficient hydrophilicity to obtain stable suspension during injection in the circulation. Among different lipophilic carriers, oilin-water emulsions can provide both a highly lipophilic core encapsulating large payload of drugs and an amphiphilic surface [2].

Nanoemulsions, dispersion of oil-in-water emulsions with particle diameters below $500 \mathrm{~nm}$, are particularly interesting for drug delivery [3] due to their capacity to dissolve large quantities of hydrophobic molecules and their ability to protect encapsulated molecules from degradation and/or enzymatic hydrolysis. They are used for instance in parenteral delivery [4]. In addition, emulsions can be tuned to modulate the delivery and achieve sustained long-term delivery on a targeted site or improve drug safety and resistance [5,6]. Contrary to larger emulsions, nanosized emulsions do not sediment nor cream [3] and present a larger surface area that can be used to functionalize them with for example, imaging agents, specific targeting molecules, etc. [7]. Although very promising, there is still a lot to investigate about controlling nanosized emulsions as compared with larger micronsized emulsions and about interactions of these objects with biological media and cells.

Among many others, the stability of nanoemulsions, for example, maintaining of physiochemical properties of the particles without adsorption of proteins or loss 
of active coating reagent, in biological conditions can still be an issue. Stabilizing emulsions can be achieved by modifying the surfactants quantities and composition but recently another way has been investigated: assembling nanoparticles coatings on the surface of these lipid-based colloids using Pickering emulsions or colloidosomes [8-11].

Another important issue is to develop multifunctional carriers, for example, carriers incorporating hydrophobic drugs, imaging agents and targeting moieties $[7,12,13]$. Thus hybrid emulsions can lead to multifunctionality through both the lipophilic core, the amphiphilic interface and the inorganic nanoparticles incorporated [14]. Indeed, incorporation of inorganic and organic components within a unique object can bring synergistic combinations of different functionalities and provide means of getting both hydrophilic and hydrophobic molecules within one carrier, possibly with some remotely triggered drug release [15].

Several ways have been developed to combine organic/inorganic in a single nanovector. One example is emulsion-based lipidic particles where the oil droplets can be coated by an inorganic layer, either continuous or made by the assembly of nanoparticles or inorganic particles can be incorporated within the lipophilic core of the vector. Adding an inorganic layer to oil droplets, for example, a silica shell as developed by Jakmola $e t$ al. [1], enables obtention of vectors that combine in a nontoxic carrier the loading ability of the oil core for hydrophobic molecules with tagging of silica with contrasting agents. In addition, the silica layer enables easy tuning of the surface charge and fluorescence while providing a protective surrounding for the encapsulated drug. In another approach, inorganic nanoparticles can coat lipid-based colloids $[9,10,16]$. It corresponds globally to the first step of synthesis of colloidosomes, structures that have been highly investigated in terms of targeted drug delivery/controlled release [11]. This approach uses nanoparticles to stabilize the interface of emulsion droplets for example but also provides a mean to modulate drug release from the carrier by enhancing physical stability of the particles, influencing release kinetics, stabilizing the encapsulated active compound against chemical degradation or controlling molecular transport of the vector. Finally, inorganic nanoparticles can be incorporated within the lipidic core $[7,14,17,18]$. Metallic nanoparticles are often used, such as iron oxide nanocrystals that act as MRI agents, magnetic nanoparticles that provide a mean of manipulation using an external magnetic field or gold nanoparticles that grant thermal responsiveness. All these studies show that hybrid nanovectors are interesting candidates for drug delivery but a few data exist to understand the influence that can arise from encap- sulating inorganic particles within a nanoemulsion in terms of interactions with the cells.

Combining solid nanoparticles and oil droplets thus seems an attractive approach but what of the influence of encapsulating solid nanoparticles within an oil droplet, not only at the outer interface but inside the droplet, on the response of cells exposed to such object. Can the encapsulation of solid particles within these soft capsules modulate cellular recognition of the particles and their subsequent intracellular fate?

Recent studies hinted that mechanical properties of the carriers can affect the entry mode inside the cells [19-24], thus encapsulating rigid nanoparticles in soft droplets might be a way to avoid endocytosis and subsequent lysosome end-point or bring another way of entry of the particles [21,25]. However, the intracellular fate of nanoemulsion is yet poorly determined while it would bring important information to help optimize intracellular targeting.

In the current study, we developed a multifunctional platform based on oil-in-water emulsions. These nanovehicles $(360 \mathrm{~nm})$ are composed of an edible oil core stabilized by a biocompatible surfactant and encapsulate hydrophobic-functionalized silica nanoparticles $(60 \mathrm{~nm})$. The concept is depicted in Figure 1. The silica nanoparticles were rendered fluorescent by covalent grafting to use fluorescence microscopy to track the particles during cell studies. After characterization, the particles were incubated with model epithelial cells HeLa to determine the effect of solid particles encapsulation within an oil droplet on their interaction with the cells, their internalization pathway and subsequent intracellular fate as compared with free solid nanoparticles.

\section{Methods}

Synthesis \& characterization of particles Hydrophobic fluorescent silica nanoparticles

Fluorescent silica nanoparticles were synthesized by solgel process as previously described [26] leading to particles called SiNP. Finally, particles were rendered hydrophobic by covalent grafting of TMS (trimethylsilane) using the procedure described by Tolnai et al. [27]. These hydrophobic particles are later on called SiNP-TMS.

\section{Emulsions}

Hydrophobic SiNP-TMS particles suspended in ethanol were dispersed in soybean oil by sonication at a concentration of $3 \% \mathrm{w} / \mathrm{w}$ and ethanol evaporated prior to emulsification. Emulsions were obtained by progressively mixing in a mortar with a pestle the oil phase to an aqueous phase consisting of Pluronic F68 15\%w/w as a surfactant and alginate solution $2 \%$ as a viscosifier. This initial emulsion (crude emulsion obtained directly after emulsification) was characterized by 


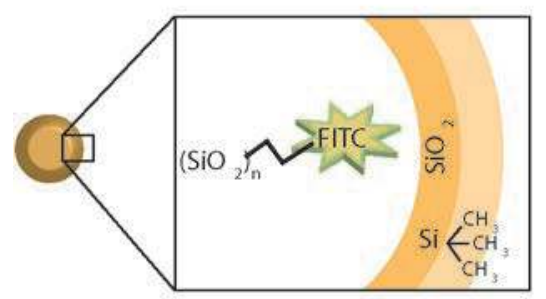

Hydrophobic fluorescent silica NPs

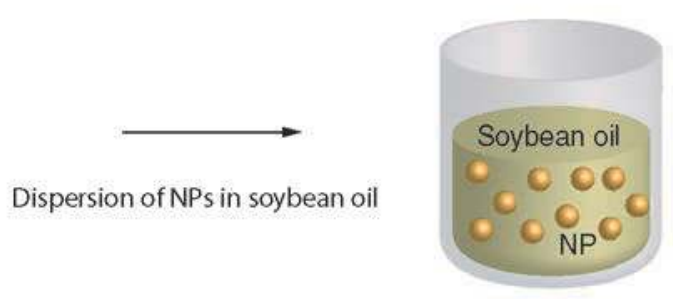

(1)

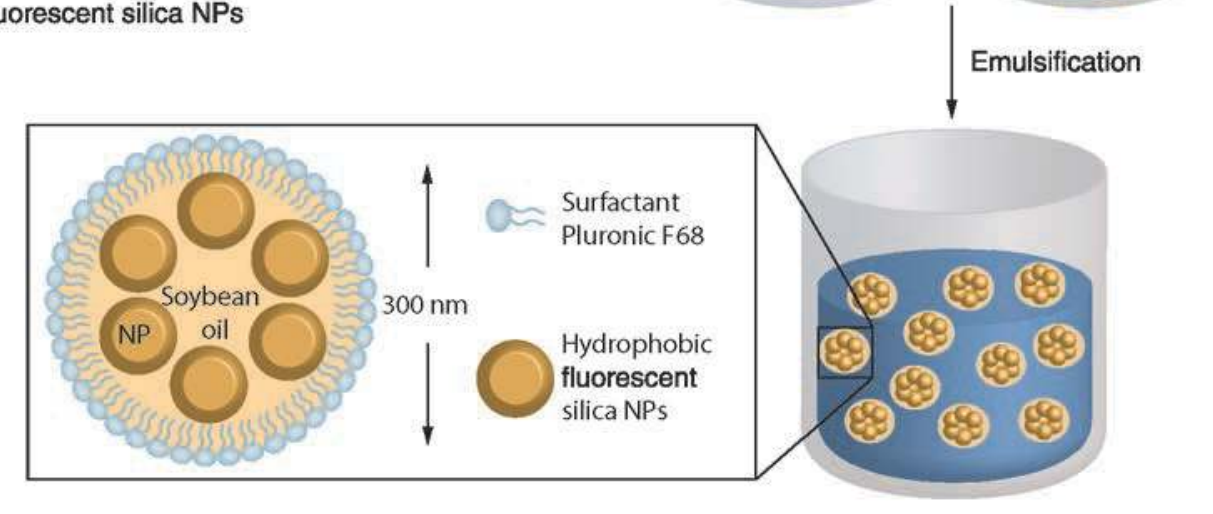

Figure 1. Scheme of fabrication steps of NE@SiNP-TMS: functionalization of silica nanoparticles (fluorescence, hydrophobic surface), dispersion within soybean oil prior to emulsification in presence of surfactant Pluronic F68.

optical microscopy. Decantation after dilution of the Pluronic F68 to 1\%w/w and oil phase to 5\%w/w led to separation of nanometric droplets from droplets with diameters above $1 \mu \mathrm{m}$, creaming process was left for several days to get a homogenous population of nanoemulsion in the aqueous phase, as checked by dynamic light scattering (DLS).

\section{Characterization}

Zetasizer Nanosizer ZS (Malvern Instruments Ltd., UK) was used to determine hydrodynamic diameter of the particles in buffer and in complete culture medium at $20^{\circ} \mathrm{C}$ using DLS. Measurements were collected at $173^{\circ}$ scattering angle. Zeta potential was measured in $10 \mathrm{mM} \mathrm{KCl}$. Concentration of the emulsions and particles was determined by weighing the suspension after water evaporation. The yield of solid particles encapsulation was determined by fluorescence spectroscopy. Spectra of the emulsion NE@SiNP-TMS and of a suspension of SiNP-TMS at a concentration corresponding to 3 w\% of NE@SiNP-TMS were recorded on a spectrofluorimeter Agilent Cary Eclipse with excitation at $485 \mathrm{~nm}$ and maximum intensity read at $515 \mathrm{~nm}$. The yield was calculated as the ratio of the intensity of fluorescence in NE@SiNP-TMS divided by the intensity of the suspension of SiNP-TMS.

\section{Cell culture \& observation}

HeLa cells (ECACC) were cultured in DMEM (Dulbecco's Modified Eagle Medium, Life Technologies) supplemented with 10\% FBS (Life technologies), 1\% glutamine and $0.7 \%$ Pen/Strep (Life technologies). Cells were used at passage 10-20. When reaching approximately $70 \%$ confluence, cells were detached with Trypsin (TrypLE Express Enzyme 1X, Life technologies) and incubated on sterile glass coverslips at a density of approximately $12,000 \mathrm{cells} / \mathrm{cm}^{2}$ overnight before incubation with particles. For observation cells were fixed using PFA (Delta Microscopy) at $4 \%$ for $20 \mathrm{~min}$ at room temperature, following by nucleus staining using Hoechst 33342 (Sigma-Aldrich) at $1 \mu \mathrm{g} \cdot \mathrm{ml}^{-1}$ for $30 \mathrm{~min}$.

\section{Internalization}

Time evolution

Cells were incubated with $0.05 \mathrm{mg} \cdot \mathrm{ml}^{-1}$ particles (emulsions@SiNP-TMS, SiNP-TMS or SiNP) in complete culture medium and fixed with $4 \%$ paraformaldehyde in PBS at different exposure times from $30 \mathrm{~min}$ to $72 \mathrm{~h}$ before observation by fluorescence microscopy. Analysis of the images to quantify nanoparticles internalization was done using CellProfiler software [28] to measure intensity of the green fluorescence channel in the different cells manually identified. Analysis was done on at least 30 cells per condition and time point.

\section{Pathway}

Inhibition of internalization was used to determine how particles entered the cells. Incubation of cells with particles at $4^{\circ} \mathrm{C}$ for $2-6 \mathrm{~h}$ was used to determine 
whether the internalization was active or passive. Incubation with monodansylcadaverine (Sigma-Aldrich, $50 \mu \mathrm{M}, 3 \mathrm{~h}$ ) prior to particles exposure was used to inhibit clathrin-mediated endocytosis.

\section{Intracellular localization}

Cells were incubated with particles and subsequently observed either by confocal fluorescence microscopy or transmission electron microscopy (TEM). Samples for TEM where fixed with $2.5 \%$ glutaraldehyde, postfixed with osmium tetraoxide and included in araldite resin before preparing thin $70 \mathrm{~nm}$ section stained with lead citrate and uranyl acetate for observation. TEM observation where done on a Philips EM120 microscope operating at $120 \mathrm{kV}$. Endosome labeling was done using DeepRed Lysotracker (Life Technologies) incubated during the last hour of particles exposure at $50 \mathrm{nM}$ prior to cell fixation with PFA $4 \%$ and imaged with a Zeiss confocal microscope. Colocalization was estimated by analyzing the confocal images with CellProfiler software adapting the available colocalization pipeline.

\section{Toxicity}

Metabolic activity was evaluated using the resazurin assay. Cells were exposed to $0.05 \mathrm{mg} \cdot \mathrm{ml}^{-1}$ particles for $24 \mathrm{~h}, 72 \mathrm{~h}$ or 7 days and concentration dependence was estimated after $24 \mathrm{~h}$ of incubation with particles concentrations ranging from 0.05 to $0.2 \mathrm{mg} \cdot \mathrm{ml}^{-1}$.

Viability of the cells exposed to $0.05 \mathrm{mg} \cdot \mathrm{ml}^{-1}$ nanoparticle concentration was evaluated using Live/ dead detection kit (Life Technologies).

\section{Microscopy}

Confocal microscopy observations of fixed cells were performed on a Zeiss LSM 710 microscope. Diode laser $405 \mathrm{~nm}$ was used to excite Hoechst 33342, argon laser $488 \mathrm{~nm}$ for FITC, helium laser $633 \mathrm{~nm}$ for Lysotracker Deep Red. Emission was detected between 430 and $540 \mathrm{~nm}$ for Hoechst 33342, 485-540 nm for FITC and 640-790 for Lysotracker. Acquisition was made in channel-separated mode with a line averaging of 8 . As we have chosen to set a constant mass concentration of particles, it resulted in a difference in terms of number of particles. Indeed, solid particles suspension of $0.05 \mathrm{mg} \cdot \mathrm{ml}^{-1}$ corresponds initially to approximately 5 million particles/cell whereas NE@SiNP-TMS at the same mass concentration correspond to approximately 100-times less particles/cell. To account for this difference, laser intensity was doubled for NE@SiNP-TMS compared with SiNP/SiNP-TMS.

Transmission electron microscopy: EFTEM \& EELS Unstained $60 \mathrm{~nm}$ ultrathin sections of HeLa cells exposed to NE@SiNP-TMS were used for qualitative element analysis. Electron energy loss spectroscopy and electron sepctroscopic imaging were carried out with a JEOL 2100HC EFTEM working at $200 \mathrm{kV}$ with LaB6 filament, equipped with a postcolumn Gatan Image Filter (GIF Tridiem) and a slow scan camera $2 \mathrm{k} \times 2 \mathrm{k}$.

Cells were searched for intracellular areas with denser electron density. EEL-spectra were measured from 90 to $500 \mathrm{eV}$ at a magnification of 5000-times with the area of interest occupying most of the field. EEL-spectra measured outside of the area of interest were used as negative controls.

Electron sepctroscopic imaging was carried out using a three-window method from 70 to $170 \mathrm{eV}$ to obtain a computer-generated distribution of the element $\mathrm{Si}$ at magnification 500-times.

\section{Statistical analysis}

Data were processed first with Wilcoxon test to evaluate data distributions. Data with Gaussian distributions were validated with paired t-test, non-Gaussian distributed sets of data were evaluated with MannWhitney. Each experiment was replicated three-times on triplicates.

\section{Results}

\section{Particles synthesis}

The aim of this work was to synthesize nanometric oil droplets encapsulating solid nanoparticles. Both these objects and free nanoparticles, either pure silica or hydrophobic, were exposed to cells to determine the impact of solid particles encapsulation within an oil droplet on its interactions with cells. Fluorescent silica nanoparticles were synthesized by sol-gel process with a covalently grafted fluorophore (FITC) that enables to track the particles by fluorescence microscopy and rendered hydrophobic to enable their dispersion within the oil phase. Then emulsification was conducted to obtain nanometric droplets, using Pluronic F68 (Poloxamer 188), an amphiphilic and nonionic PEOPPO-PEO triblock copolymer, as a nontoxic surfactant to stabilize the interface [4]. Soybean oil was chosen as the lipidic core as it gives stable emulsions commonly used in pharmaceutical formulations [29].

\section{Particles characterization}

Prior to hybrid nanometric emulsion preparation, silica nanoparticles were characterized using DLS and zeta potential as resumed in Table 1. The initial silica particles have a diameter of $70 \mathrm{~nm}$ and a negative zeta potential of $-45 \mathrm{mV}$. After trimethylsilane functionalization, the hydrodynamic diameter observed in DLS is not significantly increased when measured in ethanol (however particles rapidly form aggregates in water due to hydrophobic surface functionalization) and zeta 
Table 1. Characteristics of particles, hydrodynamic diameter measured by dynamic light scattering.

\begin{tabular}{|c|c|c|c|}
\hline & $\operatorname{Diameter}^{\dagger}(\mathrm{nm})$ & Diameter $^{\ddagger}(\mathrm{nm})$ & $\zeta$ potential $^{\dagger}(\mathrm{mV})$ \\
\hline SiNP & $65 \pm 5$ & \pm 5 & $-45 \pm 2.5$ \\
\hline \multirow[t]{2}{*}{ SiNP-TMS } & $320 \pm 30$ & Aggregates $>1 \mu \mathrm{m}$ & $-10 \pm 1$ \\
\hline & Aggregates $>1 \mu \mathrm{m}$ & & \\
\hline NE@SiNP-TMS & $360 \pm 60$ & $380 \pm 60$ & $-22 \pm 3$ \\
\hline $\begin{array}{l}\text { +In water. } \\
\text { IIn complete cultur } \\
\text { Zeta potential mea }\end{array}$ & $\mathrm{KCl}$, mean $\pm \mathrm{SD}$ & & \\
\hline
\end{tabular}

potential falls down to $-10 \mathrm{mV}$. Incorporation of SiNPTMS within soybean oil does not induce aggregation in the time course of emulsion preparation. Nanoemulsions were characterized for size dispersion and surface charge. DLS shows a monodisperse population of droplets centered at $360 \mathrm{~nm}$, with a surface potential of $-22 \mathrm{mV}$ (Figure SI-1) similar to pure oil nanoemulsion. Dispersion in culture medium leads to decreased surface charge and a neutral zeta potential. Finally, we can estimate the mean number of nanoparticles per oil droplets based on the initial concentration of nanoparticles in the oil phase. Assuming that the particles are homogeneously dispersed during emulsification, we obtain a mean number of 3-5 particles per nanodroplet (SI). Encapsulation efficiency of the solid nanoparticles within the nanoemulsion was determined by fluorescence spectroscopy (Figure SI-2) and found to be approximately $30 \%$. Observing the micrometersized emulsion (Figure SI-3) shows that the particles are pref- erentially at the interface and the measurements of zeta potential indicate that they are on the inner interface as there is no influence on surface charge compared with pure oil emulsions.

\section{Toxicity}

To consider hybrid nanoemulsions for drug delivery, it is necessary to ensure that these particles do not have unwanted toxicity. To determine cytotoxicity of these particles toward model epithelial cells HeLa, metabolic activity was monitored. Metabolic activity at 0.05 $\mathrm{mg} \cdot \mathrm{ml}^{-1}$ shows that no significant toxicity follows exposure to SiNP, SiNP-TMS or NE@SiNP-TMS for up to 7 days of continuous exposure (Figure 2A). To ensure that the level of metabolic activity indeed reflected an absence of cyctotoxicity and not the presence of cells under high stress, Live/Dead assay was conducted to correlate the number of living cells to the metabolic activity measured. Figure $2 \mathrm{~B}$ shows that the number of
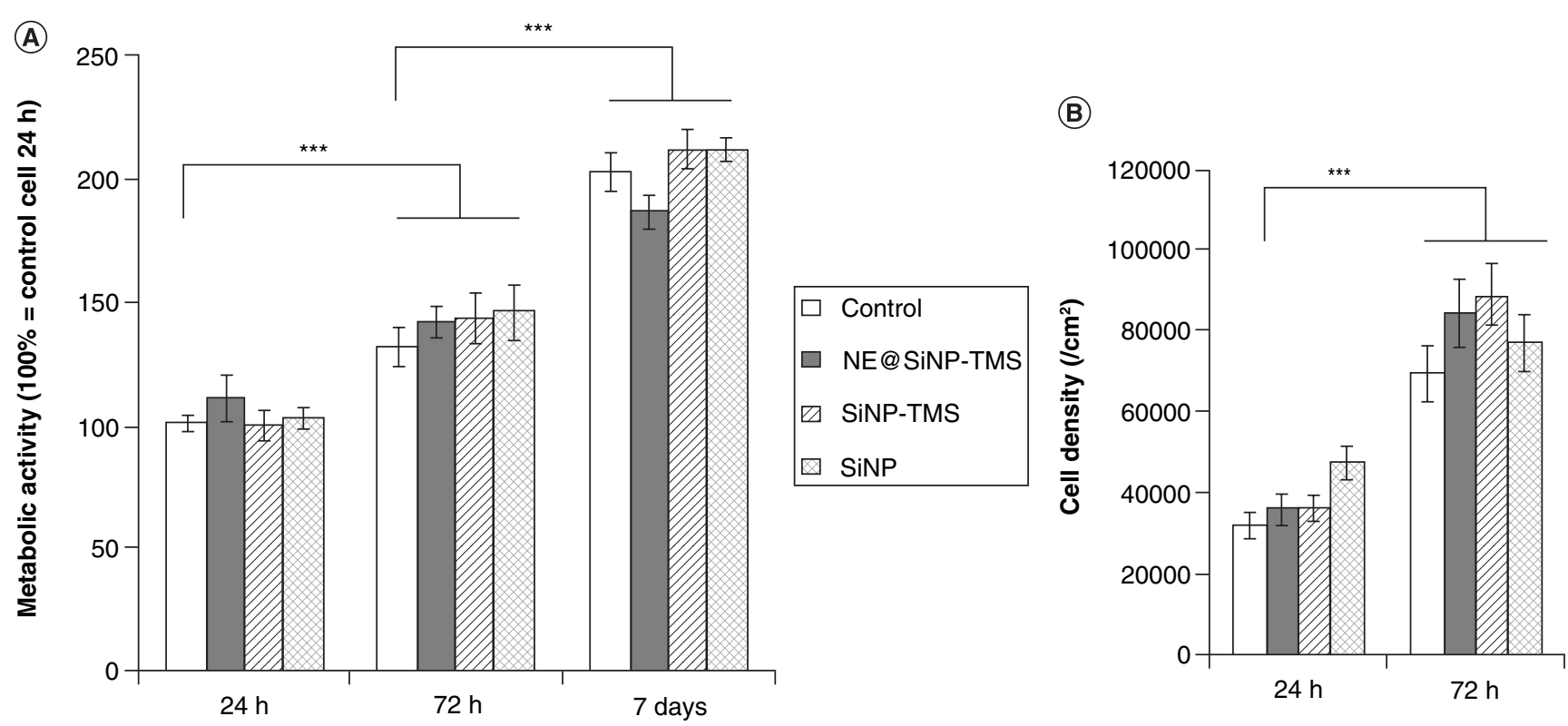

Figure 2. (A) Metabolic activity of HeLa cells exposed to $0.05 \mathrm{mg}^{-\mathrm{ml}^{-1}}$ particles suspensions, estimated by resazurin assay, normalized using control cells after $24 \mathrm{~h}$ as $100 \%$, (B) Live/dead assay of HeLa cells exposed to $0.05 \mathrm{mg}^{\mathrm{ml} \mathrm{l}^{-1}}$ particles suspensions, mean $\pm \mathrm{SD}$.

$* * *$ Statistical difference $\mathrm{p}<0.001$. 
(A) $6 \mathrm{~h}$
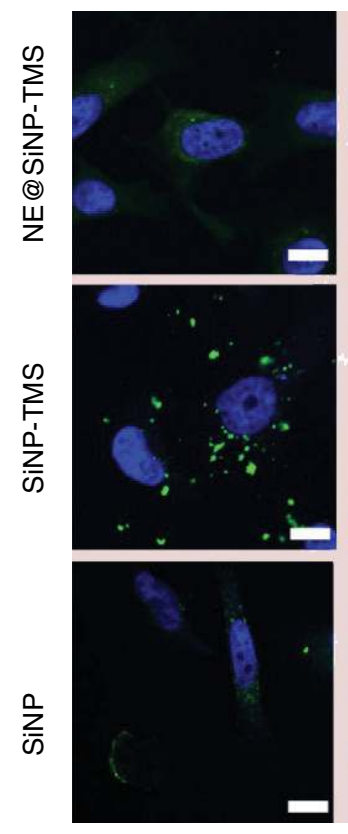

$24 \mathrm{~h}$
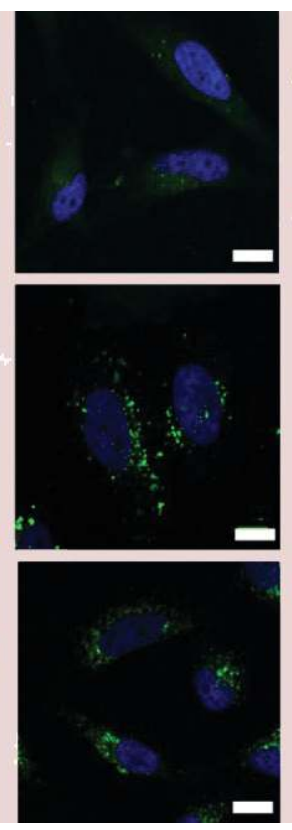

$48 \mathrm{~h}$
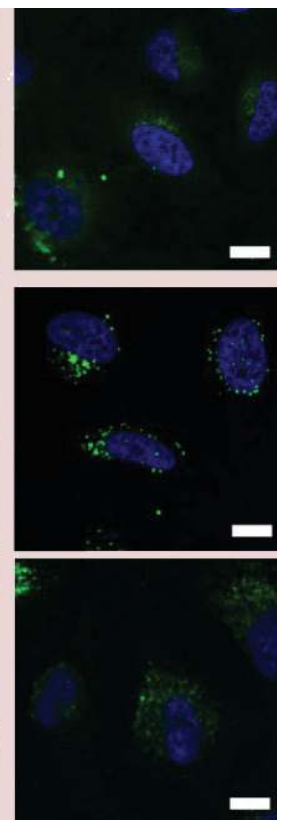

(B)

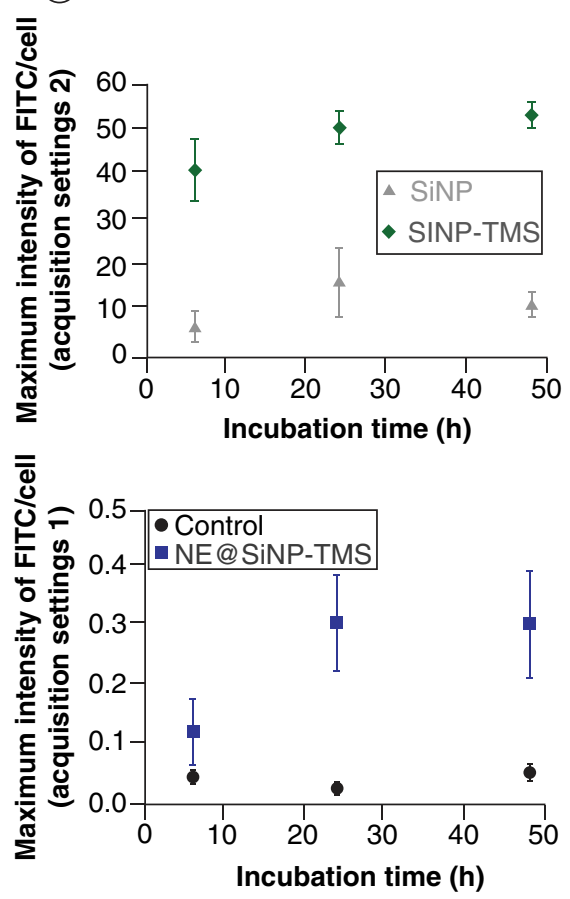

Figure 3. Kinetics of internalization of particles exposed to HeLa cells for the whole length of the assay at 0.05 mg. $\mathbf{m l}^{-1}$. (A) Confocal images, nucleus stained in blue with Hoechst 33342, FITC-labeled nanoparticles in green (NE@SiNP-TMS images were acquired with a higher laser power compared with SiNP-TMS and SiNP), scale bar = $10 \mu \mathrm{m}$. (B) Evolution of maximum intensity of internalized particles determined on fluorescence images.

living cells were consistent with the metabolic activities observed, indicating that indeed no significant toxicity could be noted for exposure to either particles at $0.05 \mathrm{mg} \cdot \mathrm{ml}^{-1}$. Concentration dependence was evaluated at $24 \mathrm{~h}$ by incubating cells with up to 20-times more particles $(1 \mathrm{mg})$ and showed no significant effect of metabolic activity of cells compared with untreated control (Figure SI-4).

\section{Internalization}

We next studied the interactions of the different particles with HeLa cells to determine if the carrier was easily internalized by cells. Here, all particles are readily internalized by HeLa cells after a short incubation period at $0.05 \mathrm{mg} \cdot \mathrm{ml}^{-1}$ (Figure 3A). Nanoparticles (SiNP or SiNP-TMS) form clusters, as observed in confocal microscopy, of very bright spots whereas nanometric emulsions NE@SiNP-TMS are more dispersed over the cytoplasm leading to a diffused fluorescent signal. This signal increases within the first $24 \mathrm{~h}$ and is stable over the period 24-48 h exposure (Figure 3B). SiNP-TMS are slower to be internalized compared with unmodified SiNP. They are initially $(6 \mathrm{~h})$ found bound to the cell membrane but hardly inside while after $24 \mathrm{~h}$ particles are internalized as observed imag- ing a z-stack in confocal microscopy (Figure SI-5) and confirmed by TEM imaging (Figure 5). In comparison, SiNP as well as NE@SiNP-TMS are already well internalized after $6 \mathrm{~h}$.

\section{Intracellular localization}

Having shown that all particles were internalized within HeLa cells, we checked the influence of the particles characteristics on their intracellular localization. To determine intracellular localization by fluorescent microscopy, lysosomes were labeled with LysoTracker, a fluorophore that specifically stains lysosomes. Few colocalization areas of the FITC signal coming from the particles in nanoemulsion NE@SiNP-TMS with lysosomes can be observed $(28 \%$ at $24 \mathrm{~h})$ whereas nanoparticles SiNP-TMS or SiNP highly colocalize with lysosomes, particularly at longer time points (52 and $65 \%$ at $24 \mathrm{~h}$, respectively) (Figure 4). To visualize more precisely intracellular localization of NE@SiNPTMS in cells after $24 \mathrm{~h}$ or $72 \mathrm{~h}$ exposure, cells were observed in TEM. Whereas SiNP-TMS were observed as aggregates in internalized vesicles inside the cells as expected (Figure 5), NE@SiNP-TMS were observed all over the cytoplasm as vesicles, with no visible deleterious effect on the cell (intact membrane, normal 
mitochondria). The particles are observed as heterogenous vesicles containing $\mathrm{Si}$ as confirmed by electron energy loss spectroscopy at $24 \mathrm{~h}$ (Figure 6). While silica particles are still observable in the internalized vesicles at $24 \mathrm{~h}$, after $72 \mathrm{~h}$ exposure all these vesicles show a more homogeneous electronic density, suggesting a degradation of the silica nanoparticles.

\section{Internalization pathways}

As observed by fluorescent or electronic microscopy, particles are differently internalized depending on whether cells are exposed to free solid nanoparticles or particles encapsulated within an emulsion droplet. To determine whether particles are internalized through an active or passive way, cells were incubated with particles at $4^{\circ} \mathrm{C}$. Decreasing the temperature to $4^{\circ} \mathrm{C}$ stops effectively internalization of SiNP and SiNP-TMS as observed with the lack of FITC-signal due to the fluorescent nanoparticles in the cells treated for $6 \mathrm{~h}$ (Figure 7). Conversely, NE@SiNP-TMS incubated at $4^{\circ} \mathrm{C}$ still gives a fluorescent signal inside the cells. This fluorescent signal is higher than the background level, but slightly lower than what can be observed when particles are incubated at $37^{\circ} \mathrm{C}$.

Furthermore, cells were incubated with particles in presence of inhibitors of clathrin-mediated endocytosis. Inhibition of clathrin-mediated endocytosis significantly reduces the internalization of nanoparticles as no more signal is observed using the same acquisition parameters (Figure 6) and only a small signal can be acquired with enhanced laser intensity and gain. In the case of NE@SiNP-TMS a decrease of fluorescent signal is observed when cells are incubated with clathrinmediated endocytosis inhibitor as compared with cells incubated with particles only.

\section{Influence of nanoemulsions on internalization of SiNP-TMS}

To check that the differences observed between NE@ SiNP-TMS and SiNP-TMS are due to the encapsulation of SiNP-TMS within oil droplets and not to the effect of free oil droplets, cells were coincubated with solid particles SiNP-TMS and nanoparticle-free nanoemulsion (Figure SI-6). We observe that the fluorescence signal observed for coincubation of solid nanoparticles and pure nanoemulsions is very similar to that obtain for cell incubated with SiNP-TMS alone.

\section{Influence of nanoemulsions \& surfactant on internalization of SiNP-TMS}

To ensure that free surfactant was not responsible for the difference of internalization observed, cells were coincubated with solid particles SiNP-TMS and $1 \%$ Pluronic F68. Figure SI-5 shows that adding the surfactant to the nanoparticles suspension dramatically decreases particles internalization as observed in fluorescence microscopy.

\section{Discussion}

In the current study, we successfully produced hybrid carriers consisting of biocompatible oil nanodroplets encapsulating solid nanoparticles. This was achieved by an emulsification method, without need for either higher concentration of surfactant than necessary for microemulsion formation nor high-energy input. This route of synthesis is thus compatible with the encapsulation of sensitive molecules, either within the solid particles or within the lipophilic oil core. It is unusual to get nanometric emulsions with such a route and can be explained by the additional stabilization of the interface brought by the nanoparticles, like in Pickering stabilization of emulsions [30]. In addition to getting access to a highly modular and adjustable nanoplateform, this vector brings interesting prospect in terms of intracellular delivery.

Soybean oil gives stable and biocompatible emulsions currently approved for pharmaceutical products that have already been tested for delivery of poorly water-soluble drugs. In addition, these emulsions have shown a potential for incorporation of high concentrations of hydrophobic drugs and enhanced release rates [29]. It thus seemed an interesting choice for the oil component of our hybrid particles. On the other side, many solid particles could have been used. Silica nanoparticles were chosen because they are by themselves very promising candidates for drug delivery and/ or imaging [31-33]. In addition, their chemistry is very versatile enabling different fictionalization and an easy way of rendering their surface hydrophobic enough to enable their encapsulation within the oil droplets and not only obtain nanoparticles on the external surface of the droplets as is done for colloidosomes.

A few studies have been conducted to investigate the potential of nanoemulsions encapsulating solid nanoparticles for drug delivery. Gianella et al. [7,14] explored the potential for cancer imaging and therapy of multifunctional soybean oil nanoemulsions encapsulating iron oxide nanoparticles. These nanoemulsions are readily taken up by different cell lines but the mechanism involved in the internalization process and subsequent intracellular localization was not investigated. Anyhow they show that such carriers are efficient for cancer imaging and therapy due to a large accumulation of the particles within the tumor. Kong et al. encapsulated porous silicon nanoparticles within giant liposomes with very encouraging results for codelivery of several drugs to obtain a synergistic effect [18]. However, contrary to our emulsion system, the liposomes 
(A)

Control
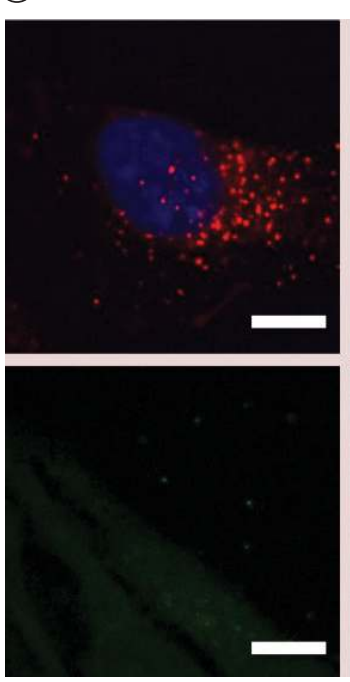

NE@SiNP-TMS
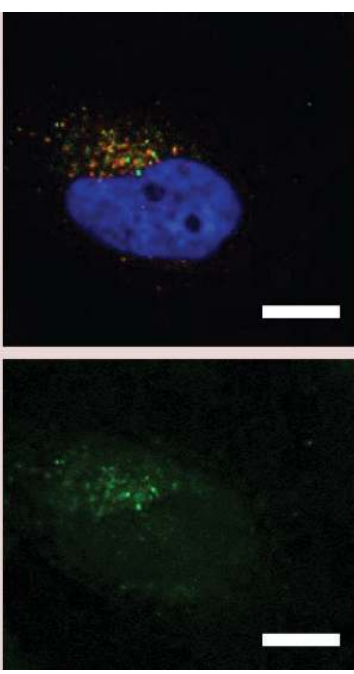

SiNP-TMS
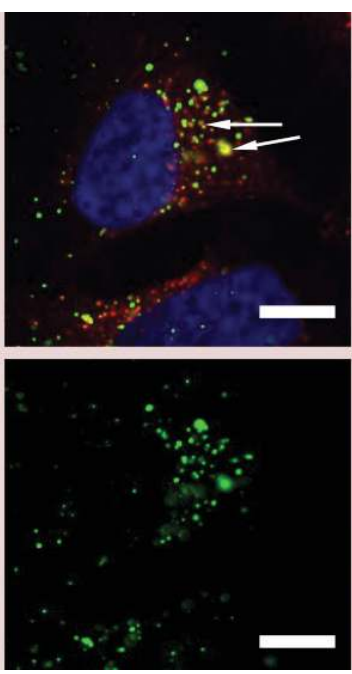

SiNP
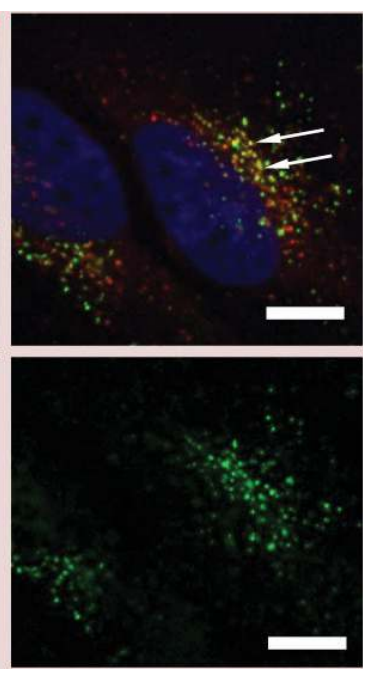

(B)

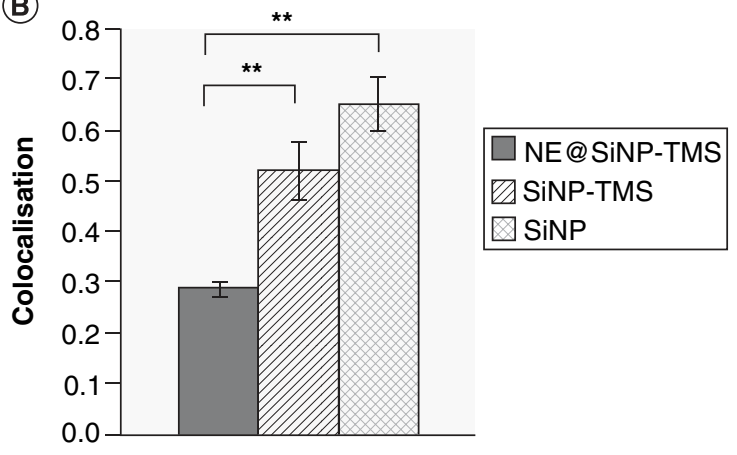

Figure 4. (A) Confocal microscopy images of internalization of particles exposed at $0.05 \mathrm{mg}^{\mathrm{m}} \mathrm{ml}^{-1}$ for $24 \mathrm{~h}$, nucleus is stained in blue with Hoechst 33342, FITC-labeled SiNP are shown in green (NE@SiNP-TMS images were acquired with a higher laser power compared with SiNP-TMS and SiNP) and Lysotracker appears red, scale bar $=10 \mu \mathrm{m}$;

(B) Colocalization of Lysotracker and fluorescence from particles, mean \pm SD. $* * \mathrm{p}<0.005$.

are fabricated using microfluidic techniques that lead to micrometersized particles.

Results of toxicity assays show that both free silica nanoparticle, either functionalized or not, or encapsulated within soybean oil nanometric droplets could be used in cell assays at relatively high doses (up to 1 $\mathrm{mg} \cdot \mathrm{ml}^{-1}$ ) and for long-term exposure (up to 7 days of continuous exposure) without noticeable adverse effect on metabolic activity. Several studies of silica nanoparticles in the size range we have used and in the same concentration range similarly report no noticeable cytotoxicity [34,35].

Even though no cytotoxicity was observed, it does not mean that the tested particles does not interact with the cells. Indeed, it appears that encapsulating solid silica nanoparticles within oil nanometric droplets interferes with their interactions with HeLa cells and modifies the intracellular localization and repartition of the particles. The observed images suggest that while free silica nanoparticles are internalized and sequestered in endocytic vesicles, nanometric emulsions are more largely dispersed within the cytosol. In addition, the fluorescent signal is lower for cells exposed to NE@SiNP-TMS compared with SiNP and SiNP-TMS. This can be explained not only because of the difference in terms of number of particles coming from setting a constant mass concentration, but also because SiNP-TMS encapsulated within nanodroplets remains separated and each drop contains only a few nanoparticles that gives a low fluorescent signal under the microscope while SiNP and SiNP-TMS are able to form large clusters that lead to highly fluorescent dots.

We thus have different cellular uptake of free silica nanoparticles, both hydrophilic SiNP and hydropho- 
bic SiNP-TMS, compared with particles encapsulated within oil nanometric droplets NE@SiNP-TMS. To rule out potential effect of free surfactant or pure oil emulsions on SiNP-TMS internalization, we coincubated those particles with either pure oil nanometric droplets and 1\% Pluronic F68. Indeed, direct cytosolic delivery of the particles could result from cell membrane permeabilization by the remaining free surfactant. Strangely, while Pluronic F68 does not prevent internalization of NE@SiNP-TMS, it limits solid particles internalization, thus the observed difference in interaction between NE@SiNP-TMS and SiNP-TMS is not due to the direct influence of free surfactant molecules. This apparent inhibition of SiNP-TMS internalization in presence of micelles of Pluronic F68 can result from either micelles or free molecules of surfactant coating the surface which modifies protein adsorption and consequently cellular internalization [36,37]. Furthermore, while studying the effect of pluronic block copolymers as biological response modifiers [38] several studies have shown that while monomers of these amphiphilic molecules can incorporate into membranes to change their microviscosity and have thus abilities to translocate across cellular membranes, these effects are not apparent anymore above the critical micellization concentration (CMC) [38]. In our study, both stock suspensions and final working solutions are at concentrations of Pluronic F68 well above the CMC $(1 \% \mathrm{w} / \mathrm{w}$ and $0.16 \% \mathrm{w} / \mathrm{w}$, respectively, $\mathrm{CMC}=0.01-0.03 \% \mathrm{w} / \mathrm{w}[4,39])$, thus it is consistent that no translocation effect is observed for SiNP-TMS.
To dismiss the potential impact of pure oil nanodroplets on internalization of prereleased SiNP-TMS, cells were coincubated with pure oil nanoemulsions and SiNP-TMS. The similarity between the signal observed for cell incubated with SiNP-TMS or coincubated with SiNP-TMS and pure oil nanoemulsions suggests that the nanoemulsion can affect nanoparticles penetration within cells only when the nanoparticles are encapsulated within the droplets and that it is not an adjuvant effect. It hence gives indirect evidence of the absence of release of the solid nanoparticles from NE@SiNPTMS. We can finally conclude that the difference of internalization observed between NE@SiNP-TMS and SiNP-TMS is due to the encapsulation of the solid nanoparticles within the oil droplets.

Several physicochemical parameters of the nanoparticles have been established as important for cellular internalization [40] and the crossing of cellular barriers [41], which can explain the differences observed between solid nanoparticles in suspension or encapsulated within oil nanodroplets. Size and surface charge have long been known to strongly influence the ability of cells to recognize and internalize nanoparticles, the smallest particles being generally more readily internalized. In addition, more recently, geometry, mechanical parameters such as rigidity/flexibility [19,21,42], elasticity [22] or deformability [23] and hydrophobicity [43] have arisen as other important features. For example, Bai et al. [43] have shown using water-soluble organic nanoparticles that the more lipophilic particles entered HeLa cells more easily, even in presence of serum proteins.
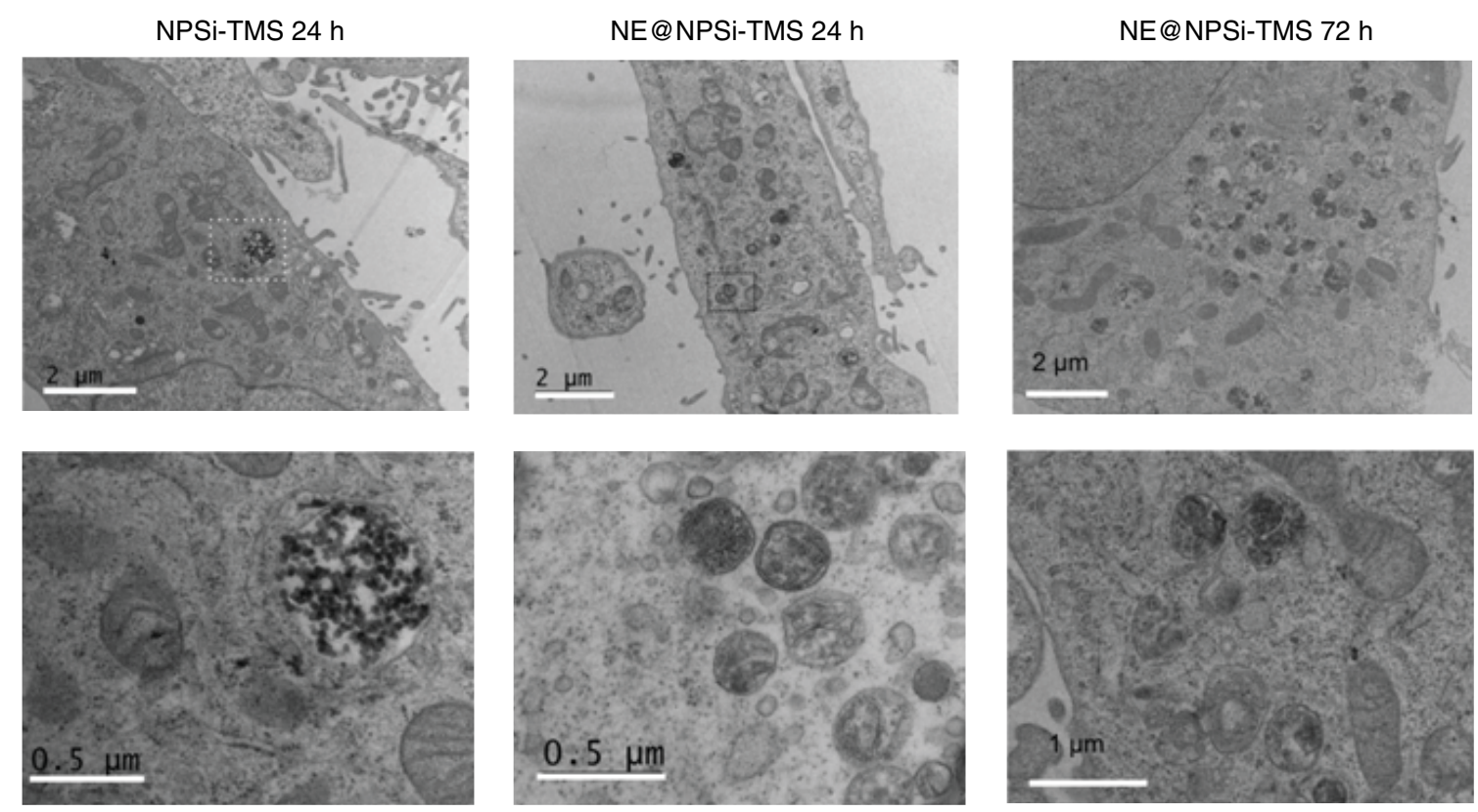

Figure 5. TEM images of HeLa cells exposed to $0.05 \mathrm{mg}^{\mathrm{m}} \mathrm{m}^{-1}$ particles NE@SiNP-TMS or SiNP-TMS for $24 \mathrm{~h}$ and $72 \mathrm{~h}$. 
(A)

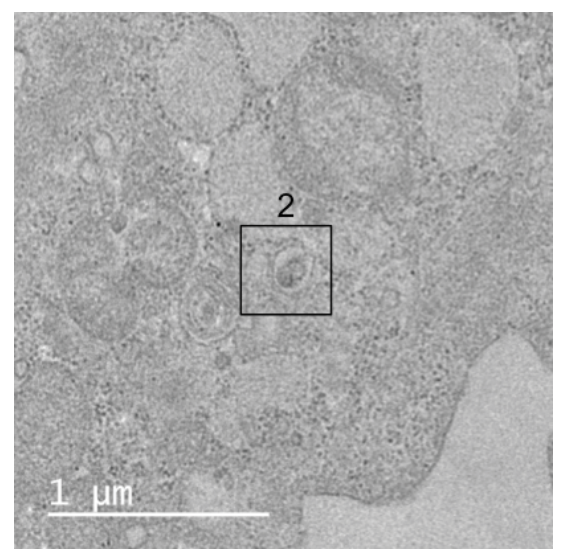

(B)

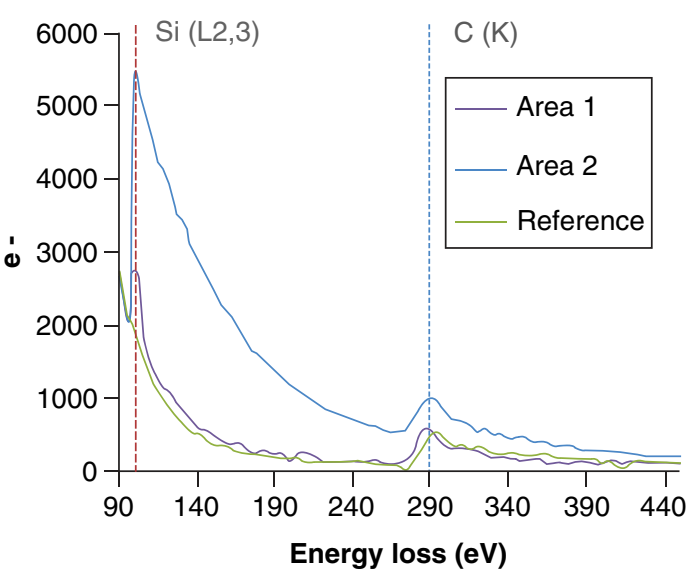

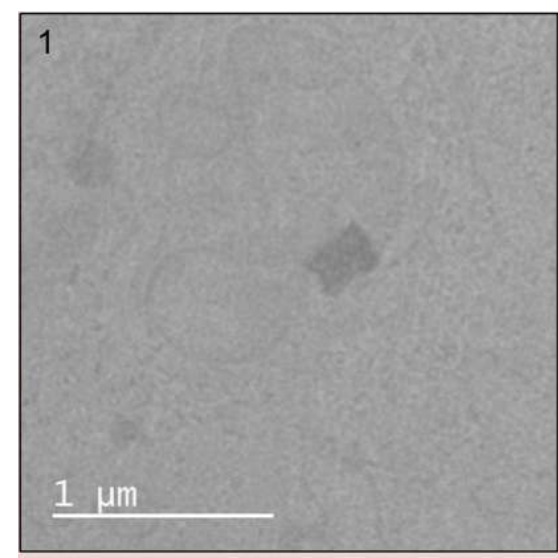

(C)

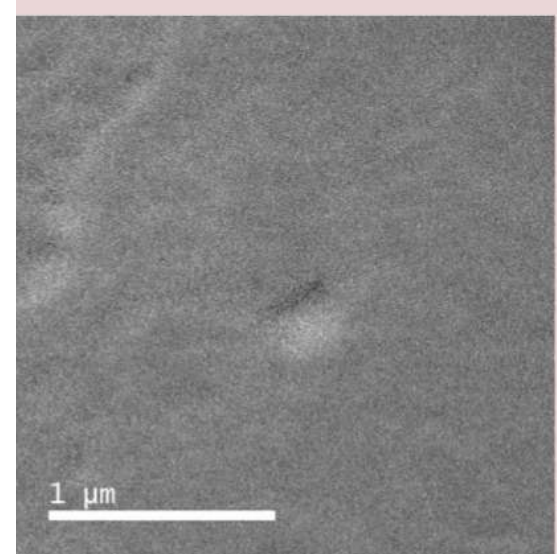

Figure 6. (A) Zero-loss EFTEM images of internalized NE@SiNP-TMS after $24 \mathrm{~h}$ exposure at $0.05 \mathrm{mg}$.ml-1 to HeLa cells, (B) EELS graph from area in the boxes defined in (A) and reference measurement in the cell but outside the area with nanoparticles, (C) computer-generated distribution of the element Si in area 1.

EELS: Electron energy loss spectroscopy.

In addition, NE@SiNP-TMS, composed of an oil core, are softer than silica nanoparticles, another characteristics that can impact internalization. Li et al. [21], in a molecular simulation study showed that soft nanoparticles might face decreased endocytosis but alternatively could enter the cell by penetration mechanisms without creation of internalization vesicles. This seems in agreement with our results concerning the internalization route followed by either particles. Indeed, solid nanoparticles are internalized by an active clathrin-mediated endocytosis, commonly encountered in this size-range [44,45], whereas nanometric emulsions NE@SiNP-TMS are undergoing endocytosis but also partial internalization by a nonenergetic pathway.

This phenomenon of membrane translocation has been observed by other investigators in various cell lines and for several kind of particles $[25,46]$. Even though model and theoretical studies have not yet been able to conclude as to which mechanism is involved, it seems that size and hydrophobicity are important parameters to be considered [47], and that a simple diffusive process is unlikely to be responsible for transmembrane transport of nanoparticles below $100 \mathrm{~nm}$ [48]. It also appears that the arrangement and density of the ligand used to help membrane penetration has a major influence on the ability of a particle to indeed cross the membrane [49]. Thus having a liquid interface brought by the oil droplets might help setting an arrangement of surface functional groups that favors membrane penetration.

All our results combined thus lead to the hypothesis that the difference of cellular uptake in HeLa and subsequent intracellular localization of the particles is a result of mechanical properties and/or hydrophobicity brought by the nanoemulsion. Finally, oil droplets provide another characteristics that can be exploited in drug delivery: softer particles have been shown to have longer circulation times in vivo [22,23]. Encapsulating solid nanoparticles within nanometric biocompatible 

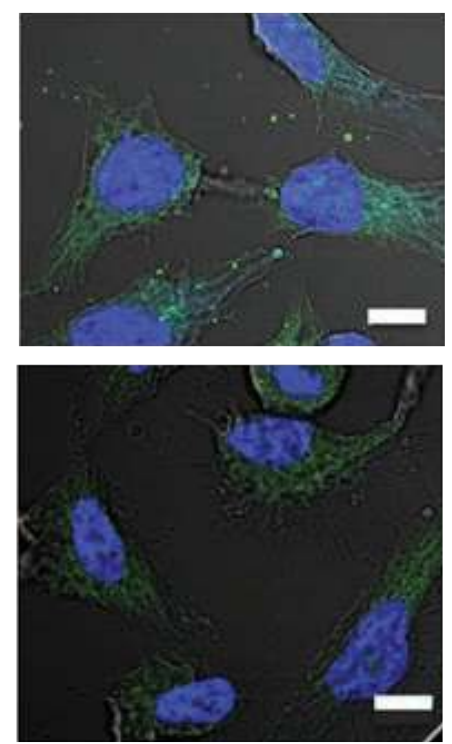

Inhibition of energy-driven process

Inhibition of clathrin-mediated endocytosis
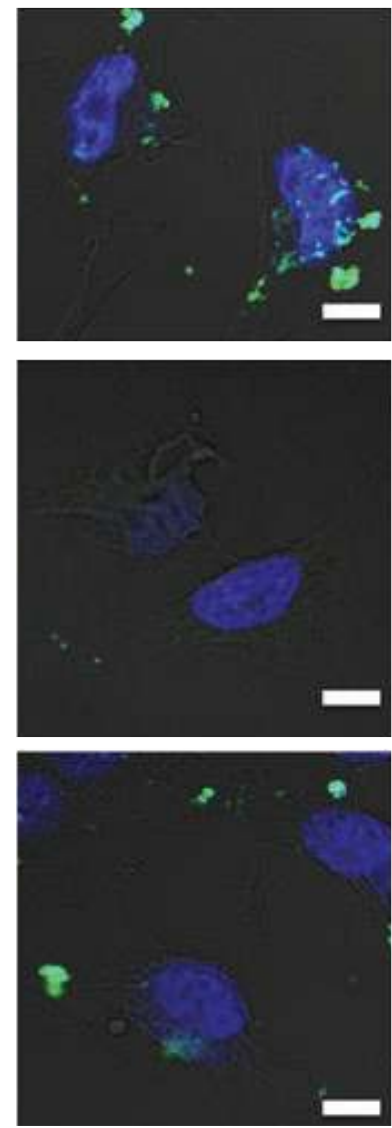

SiNP
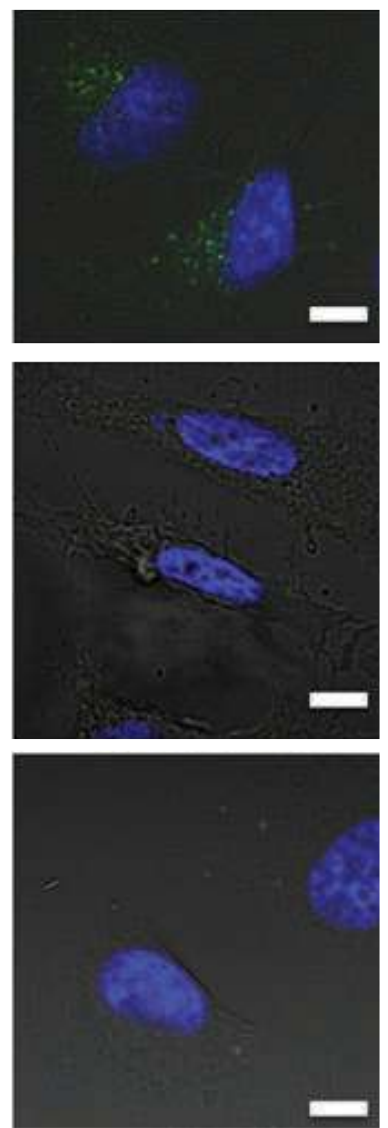

Figure 7. Internalization of particles exposed at $0.05 \mathrm{mg} \cdot \mathrm{ml}^{-1}$ for $6 \mathrm{~h}$, with inhibition of energy-driven process (incubation at $4^{\circ} \mathrm{C}$ ) and with inhibition of clathrin-mediated endocytosis, composite of transmission images in dark gray and fluorescence channels (green $=$ FITC, blue $=$ nuclei), scale bar $=10 \mu \mathrm{m}$.

oil droplets could thus become another way of modulating internalization pathway of solid nanoparticles in addition to providing an easy mean of codelivering high payloads of hydrophobic and hydrophilic/ imaging molecules within the cell.

\section{Conclusion}

We developed a nanometric vector composed of solid nanoparticles encapsulated in a soybean oil droplet with a synthesis route compatible with encapsulation of sensitive lipophilic molecules. Our results show that such a hybrid vector can be used for efficient intracellular delivery. Interestingly, we observed some nonenergy-driven internalization and a noticeable impact on particles intracellular localization when incubated with Hela cells. For the moment, we cannot yet elucidate the exact mechanism of the observed effect but we can suggest that both low rigidity and hydrophobicity of the vector might play an important role on the interaction of the nanoparticle with the cell membrane and its subsequent internalization.

\section{Supplementary data}

To view the supplementary data that accompany this paper please visit the journal website at: www.futuremedicine.com/ doi/full/10.2217/nnm-2016-0074

\section{Acknowledgements}

We thank P Rostaing (IBENS) for his help with TEM (samples conditioning and observations).

\section{Financial \& competing interests disclosure}

This work has received support of the Région lle de France (DIM Nano-K PME), the CNRS (Prise de Risque PhysiqueChimie- Biologie), "Institut Pierre-Gilles de Gennes" ("Investissements d'avenir": ANR-10-IDEX-0001-02 PSL and Equipement d'excellence: ANR-10-EQPX-34). The authors have no other relevant affiliations or financial involvement with any organization or entity with a financial interest in or financial conflict with the subject matter or materials discussed in the manuscript apart from those disclosed.

No writing assistance was utilized in the production of this manuscript. 
Ethical conduct of research

The authors state that they have obtained appropriate institutional review board approval or have followed the principles outlined in the Declaration of Helsinki for all human or animal experimental investigations. In addition, for investigations involving human subjects, informed consent has been obtained from the participants involved.

\section{Executive summary}

- We developed a biocompatible combined hydrophilic/lipophilic nanocomposite by an easy procedure.

- Incorporation of silica nanoparticles within the oil phase prior to emulsification favors obtaining of nanometric droplets without need for enhanced surfactant concentration nor high-energy methods.

- The synthesized nanocomposite shows no cytotoxicity in HeLa cells for high concentrations (up to $1 \mathrm{mg}^{-\mathrm{ml}^{-1} \text { ) }}$ and long-term exposure (up to 7 days continuous exposure).

- Encapsulating solid silica nanoparticles in oil droplets strongly impact the intracellular localization in HeLa cells with a significant decrease of lysosome colocalization.

- Encapsulating solid silica nanoparticles in oil droplets have no significant impact on uptake kinetics in HeLa cells.

- Nanometric emulsions containing silica nanoparticles can partially enter the cells by a nonenergy-driven process without apparent membrane degradation.

- This kind of vectors could be tested for intracellular codelivery of lipophilic molecules easily encapsulatable within the soybean oil core and hydrophilic drugs or imaging agents incorporated within the solid particles.

\section{References}

Papers of special note have been highlighted as: $\bullet$ of interest; $\bullet$ of considerable interest

1 Jakhmola A, Vecchione R, Guarnieri D et al. Bioinspired oil core/silica shell nanocarriers with tunable and multimodal functionalities. Adv. Healthc. Mater. 4(17), 2688-2689 (2015).

2 Leal-Calderon F, Schmitt V, Bibette J. Emulsion Science: Basic Principles. Springer, NY, USA (2007).

3 Lovelyn C. Current state of nanoemulsions in drug delivery. J. Biomater. Nanobiotechnol. 2(5), 626-639 (2011).

4 Wulff-Pérez M, Torcello-Gómez A, Gálvez-Ruíz MJ, MartínRodríguez A. Stability of emulsions for parenteral feeding: preparation and characterization of $\mathrm{o} / \mathrm{w}$ nanoemulsions with natural oils and Pluronic $\mathrm{f} 68$ as surfactant. Food Hydrocoll. 23(4), 1096-1102 (2009).

5 Bee S, Banerjee A, Önyüksel H. Improvement of drug safety by the use of lipid-based nanocarriers. J. Control. Release 163(1), 34-45 (2012).

- Shows that lipid-based nanocarriers are promising option for formulation of pharmaceuticals to improve treatment safety and efficacy.

6 Gupta R, Shea J, Scafe C, Shurlygina A, Rapoport N. Polymeric micelles and nanoemulsions as drug carriers: therapeutic efficacy, toxicity, and drug resistance. J. Control. Release 212, 70-77 (2015).

7 Gianella A, Jarzyna PA, Mani V et al. Multifunctional nanoemulsion platform for imaging guided therapy evaluated in experimental cancer. ACS Nano 5(6), 4422-4433 (2011).

8 Chen $\mathrm{Y}$, Gao $\mathrm{Y}$, Chen $\mathrm{H}$ et al. Engineering inorganic nanoemulsions/nanoliposomes by fluoride-silica chemistry for efficient delivery/co-delivery of hydrophobic agents. $A d v$. Funct. Mater. 22(8), 1586-1597 (2012).
9 Simovic S, Barnes TJ, Tan A, Prestidge C. Assembling nanoparticle coatings to improve the drug delivery performance of lipid based colloids. Nanoscale 4(4), 1220-1230 (2012).

- Shows that assembling nanoparticle layers at the interface of lipid colloids enhance physical stability and in the meantime can increase absorption, bioavailability and facilitate targeted delivery.

10 Simovic S, Prestidge C. Nanoparticle layers controlling drug release from emulsions. Eur. J. Pharm. Biopharm. 67(1), 39-47 (2007).

11 Rossier-Miranda FJ, Schroën CGPH, Boom RM. Colloidosomes: versatile microcapsules in perspective. Colloids Surf. A Physicochem. Eng. 343, 43-49 (2009).

12 Jabr-Milane L, van Vlerken L, Devalapally H et al. Multifunctional nanocarriers for targeted delivery of drugs and genes. J. Control. Release 130(2), 121-128 (2008).

13 Ganta S, Deshpande D, Korde A, Amiji M. A review of multifunctional nanoemulsion systems to overcome oral and CNS drug delivery barriers. Mol. Membr. Biol. 27(7), 260-273 (2010).

14 Jarzyna P, Skajaa T, Gianella A et al. Iron oxide core oil-inwater emulsions as a multifunctional nanoparticle platform for tumor targeting and imaging. Biomaterials 30(36), 6947-6954 (2009).

15 Liu J, Detrembleur C, Mornet S, Jérôme C, Duguet E. Design of hybrid nanovehicles for remotely triggered drug release: an overview. J. Mater. Chem. B 3(30), 6117-6147 (2015).

16 Tikekar RV, Pan Y, Nitin N. Fate of curcumin encapsulated in silica nanoparticle stabilized Pickering emulsion during storage and simulated digestion. Food Res. Int. 51(1), 370-377 (2013).

17 Lee JY, Carugo D, Crake C et al. Nanoparticle-loaded protein-polymer nanodroplets for improved stability and conversion efficiency in ultrasound imaging and drug delivery. Adv. Mater. 27(37), 5484-5492 (2015). 
18 Kong F, Zhang X, Zhang $\mathrm{H}$ et al. Inhibition of multidrug resistance of cancer cells by co-delivery of DNA nanostructures and drugs using porous silicon nanoparticles@giant liposomes. Adv. Funct. Mater.25(22), 3330-3340 (2015).

19 Sun J, Zhang L, Wang J et al. Tunable rigidity of (polymeric core)-(lipid shell) nanoparticles for regulated cellular uptake. Adv. Mater. 27(8), 1402-1407 (2015).

Zhang L, Feng Q, Wang J et al. Microfluidic synthesis of hybrid nanoparticles with controlled lipid layers: understanding flexibility-regulated cell-nanoparticle interaction. ACS Nano 9(10), 9912-9921 (2015).

21 Li Y, Zhang X, Cao D. Nanoparticle hardness controls the internalization pathway for drug delivery. Nanoscale 7(6), 2758-2769 (2015).

- A molecular dynamic study that simulates the effect of nanoparticle hardness on internalization efficiency and shows that soft nanoparticle tend to face frustrated endocytosis and instead use a penetration pathway.

22 Anselmo AC, Zhang M, Kumar S et al. Elasticity of nanoparticles influences their blood circulation, phagocytosis, endocytosis and targeting. ACS Nano 3, 3169-3177 (2015).

- Shows that elasticity of the nanoparticles dramatically affects their circulation time, reduces the immune system uptake and improves targeting.

Best JP, Yan Y, Caruso F. The role of particle geometry and mechanics in the biological domain. Adv. Healthc. Mater. 1(1), 35-47 (2012).

24 Ravi Theaj Prakash U, Thiagarajan P. Nanoemulsions for drug delivery through different routes. Res. Biotech. 2(3), 1-13 (2011).

25 Jiang Y, Tang R, Duncan B et al. Direct cytosolic delivery of siRNA using nanoparticle-stabilized nanocapsules. Angew. Chem. Int. Ed. Engl. 53, 1-6 (2014).

26 Quignard S, Coradin T, Mosser G, Boissière M. Long-term fate of silica nanoparticles interacting with human dermal fibroblasts. Biomaterials 33(17). 4431-4442 (2012).

27 Tolnai G, Csempesz F, Ka E, Ramsden JJ, Ho Z. Preparation and characterization of surface-modified silica-nanoparticles. Langmuir 12(19), 2683-2687 (2001).

28 Lamprecht M, Sabatini D, Carpenter A. CellProfilerTM: free, versatile software for automated biological image analysis. Biotechniques 42 (1), 71-77 (2007)

29 Aloisio C, Longhi MR, De Oliveira AG. Development and characterization of a biocompatible soybean oil-based microemulsion for the delivery of poorly water-soluble drugs. J. Pharm. Sci. 104(10), 3535-3543 (2015).

30 Dickinson E. Use of nanoparticles and microparticles in the formation and stabilization of food emulsions. Trends Food Sci. Technol. 24(1), 4-12 (2012)

31 Barbé C, Bartlett J, Kong L et al. Silica particles: a novel drug-delivery system. Adv. Mater. 16(21), 1959-1966 (2004).
32 Parveen S, Misra R, Sahoo SK. "Nanoparticles: a boon to drug delivery, therapeutics, diagnostics and imaging. Nanomedicine 8(2), 147-166 (2012).

33 Vivero-Escoto JL, Slowing II, Trewyn BG, Lin VS-Y. Mesoporous silica nanoparticles for intracellular controlled drug delivery. Small 6(18), 1952-1967 (2010).

34 Malvindi MA, Brunetti V, Vecchio G et al. SiO2 nanoparticles biocompatibility and their potential for gene delivery and silencing. Nanoscale 4(2), 486-495 (2012).

35 Kong W, Li H, Liu J et al. Size-dependent and real-time effect of $\mathrm{SiO} 2$ nanoparticles on a single living hela cell membrane permeability. J. Mater. Chem. B 3, 1198-1203 (2015).

36 Amiji M, Park K. Prevention of protein adsorption and platelet adhesion on surfaces by PEO / PPO / PEO triblock copolymers. Biomaterials 13(10), 682-692 (1992).

37 Sarkar B, Venugopal V, Tsianou M, Alexandridis P. Adsorption of Pluronic block copolymers on silica nanoparticles. Colloids Surf. A Physicochem. Eng. Asp. 422, 155-164 (2013).

38 Batrakova EV, Kabanov AV. Pluronic block copolymers: evolution of drug delivery concept from inert nanocarriers to biological response modifiers. J. Control. Release 130(2), 98-106 (2008).

- Highlights the potential of pluronic block copolymers in terms of biological response modifications, for example, sensitization of tumors, enhancement of drug transport across biological barriers.

39 Pitto-barry A, Barry NPE. Pluronic block-copolymers in medicine: from chemical and biological versatility to rationalisation and clinical advances. Polym. Chem. 5(10), 3291-3297 (2014).

40 Canton I, Battaglia G. Endocytosis at the nanoscale. Chem. Soc. Rev. 41, 2718-2739 (2012).

41 Barua S, Mitragotri S. Challenges associated with penetration of nanoparticles across cell and tissue barriers: a review of current status and future prospects. Nano Today 9(2), 223-243 (2014).

42 Zhang L, Feng Q, Wang J et al. Microfluidic synthesis of hybrid nanoparticles with controlled lipid layers: understanding flexibility-regulated cell-nanoparticle interaction. ACS Nano 10, 9912-9921 (2015).

43 Bai Y, Xing H, Wu P et al. Chemical control over cellular uptake of organic nanoparticles by fine tuning surface functional groups. ACS Nano 9(10), 10227-10236 (2015).

44 Zhu J, Liao L, Zhu L et al. Size-dependent cellular uptake efficiency, mechanism, and cytotoxicity of silica nanoparticles toward HeLa cells. Talanta 107, 408-415 (2013).

45 Hao N, Li L, Zhang Q et al. The shape effect of PEGylated mesoporous silica nanoparticles on cellular uptake pathway in Hela cells. Microporous Mesoporous Mater. 162, 14-23 (2012).

46 Li Y, Wu Z, He W et al. Globular protein-coated paclitaxel nanosuspensions: interaction mechanism, direct cytosolic delivery, and significant improvement in pharmacokinetics. Mol. Pharm. 12(5), 1485-1500 (2015). 
47 Li Y, Chen X, Gu N. Computational investigation of interaction between nanoparticles and membranes: hydrophobic/hydrophilic effect. J. Phys. Chem. B 112, 16647-16653 (2008).

48 Banerji SK, Hayes MA. Examination of nonendocytotic bulk transport of nanoparticles across phospholipid membranes.

Langmuir 23, 3305-3313 (2007).
49 Verma A, Stellacci F. Effect of surface properties on nanoparticle-cell interactions. Small 6(1), 12-21 (2010).

- Overview of the different physicochemical parameters that affect nanoparticles interactions with cells. 